\title{
Organic Surface Modification and Analysis of Titania Nanoparticles for Self- Assembly in Multiple Layers
}

\author{
Steffi Rades ${ }^{1}$, Vasile-Dan Hodoroaba ${ }^{1}$, Erik Ortel ${ }^{1}$, Thomas Wirth ${ }^{1}$, Patrizia Borghetti ${ }^{2}$, Sarai \\ García $^{3}$, Estibaliz Gómez ${ }^{3}$, Miren Blanco ${ }^{3}$, Gabriele Alberto ${ }^{4}$ and Gianmario Martra ${ }^{4}$. \\ 1. Federal Institute for Materials Research and Testing (BAM), Division Surface Analysis and \\ Interfacial Chemistry, Berlin, Germany. \\ 2. Université Pierre et Marie Curie (UPMC), Institut des Nanosciences de Paris (insp), Paris, France. \\ 3. IK4-TEKNIKER, Chemistry of the Surface Unit, Eibar, Spain. \\ 4. University of Torino, Department of Chemistry, Torino, Italy.
}

The characteristics of $\mathrm{TiO}_{2}$ coatings can greatly influence their final performance. In the EU/FP7 project SETNanoMetro (http://www.setnanometro.eu/), different deposition procedures are being set for applying films of $\mathrm{TiO}_{2}$ nanoparticles (NPs) with defined and homogenous thickness on supports of interest for large-scale applications [1]. The selected substrates are the following: (i) silica glasses for photocatalytic measurements, (ii) Ti-alloys for orthopedic and/or dental prostheses, and for cell cultures, and (iii) conductive glasses (e.g. Fluorine doped Tin Oxide, FTO) for dye-sensitized solar cells (DSSC). From the different film deposition procedures, self-assembly [2] of $\mathrm{TiO}_{2} \mathrm{NPs}$ in multiple layers was selected for systematic characterization. For this, surface modification of the substrate and of $\mathrm{TiO}_{2}$ NPs with e.g. silane coupling agents [3] is a prerequisite.

A set of $\mathrm{TiO}_{2}$ NPs has been produced by reaction with either (3-aminopropyl)phosphonic acid (APPA) or (3-aminopropyl)triethoxysilane (APTS) in order to functionalize the surface with free amino-groups (see Fig. 1). Then, the complementary NP set can be obtained from an aliquot of the first one, through the conversion of free surface amino-groups to aldehydes by reaction with glutaraldehyde (Gluta). A proper approach for the functionalization of several types of $\mathrm{TiO}_{2} \mathrm{NPs}$ differing in size, shape and specific surface area was developed, see SEM micrograph in Fig. 2 left as an example of a type of NPs with truncated bipyramidal shape [4]. EDX, XPS, AES and ToF-SIMS spectra have been collected and analyzed to evaluate the functionalization of the different types of $\mathrm{TiO}_{2}$ NPs.

Qualitative investigation of the bulk elemental composition by means of EDX of $\mathrm{TiO}_{2} \mathrm{NPs}_{\text {identified }}$ quickly phosphorus in case of APPA treated NP sample sets (see Fig. 2 middle as an example) and silicon in case of APTS treated sample sets. Hence, functionalization of the different NPs types with APPA and APTS, respectively, is indicated. The detection of nitrogen as a marker element originating from APPA and APTES, respectively, and its discrimination from the intense Ti L peak was not possible, due to the Ti L - N K line interference in EDX spectra. AES as a surface sensitive method allows analyzing elemental and, sometimes, chemical state of the first few nanometers from the top of (individual) NPs. The P KLL spectra of bare NPs show no evidence of phosphorus, whereas $\mathrm{P}$ is clearly detectable after functionalization of samples with APPA (Fig. 2 right). This indicates the presence of APPA molecules on the surface of the $\mathrm{TiO}_{2}$ NPs. In contrast to these results, AES spectra of the NPs treated in a first step with APTS as well as in a second step with Gluta show a Si peak - as expected; however, even the bare NPs contain a few amount of Si at the NP surface. Furthermore, peak intensities and shape of functionalized NPs (first as well as second step) are of heterogenous nature. Possible explanation might be slight cross-contamination during synthesis. The strong interferences between N KLL and Ti LMM Auger peaks prevent from obtaining reliable information on nitrogen and therefore, to assess the NP functionalization with APPA or APTS. Gluta is constituted only of $\mathrm{C}, \mathrm{O}$ and $\mathrm{H}$, hence, it is not possible to obtain specific information on the additional presence of these molecules after reaction with APPA or APTS 
functionalized NPs. However, with XPS the presence of glutaraldehyde is observed in spectra of the near-surface region of the NPs. ToF-SIMS measurements were finally performed on the top-surface of the NPs. As a result, on the surface even of the bare samples secondary Si-containing ions are detected, which is in agreement with AES results. Though, their intensities after functionalization with APTS are raised, which is expected. Furthermore, secondary ions containing nitrogen of sample treated with APTS and subsequently with glutaraldehyde, have been detected, giving further indication of successful functionalization [5].

\section{References:}

[1] E Ortel et al, Surf. Interface Anal. 48 (2016) p. 664.

[2] P Pallavicini et al, Journal of Colloid and Interface Science 332 (2009), p. 432.

[3] J. Zhao et al, Colloids and Surfaces A: Physicochem. Eng. Aspects 413 (2012), p. 273.

[4] C Deiana et al., Phys. Chem. Chem. Phys. 15 (2013), p. 307.

[5] This project has received funding from the European Union's Seventh Framework Programme under grant agreement no. 604577 (SETNanoMetro).

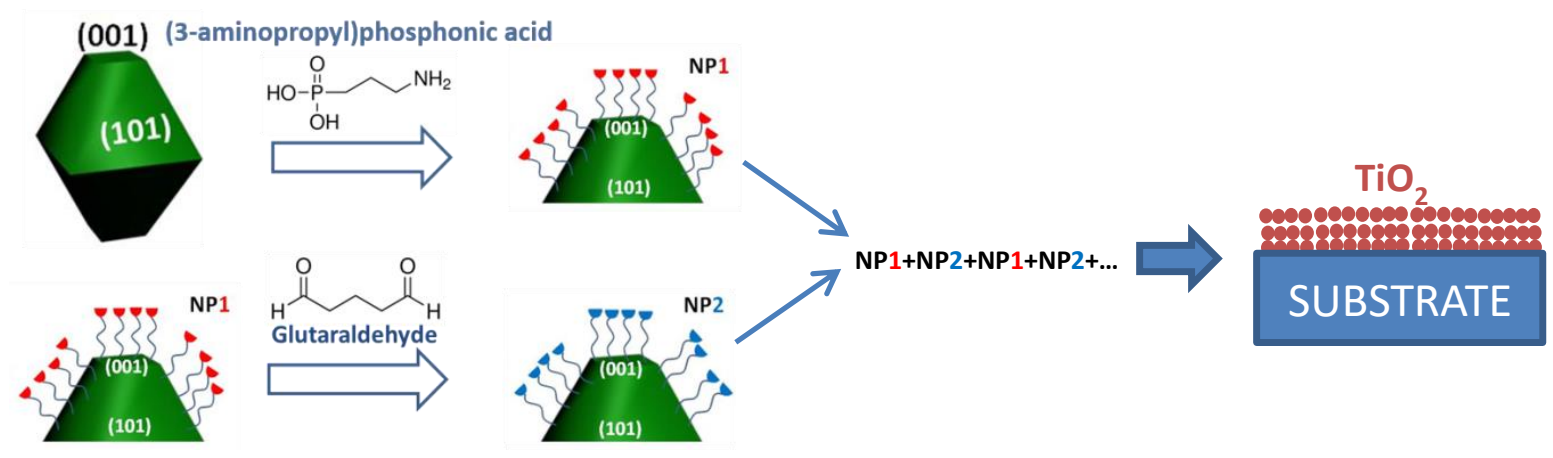

Figure 1. Strategy for preparation of two complementary sets of $\mathrm{TiO}_{2}$ NPs for film formation via layer-by-layer deposition of functionalized NPs.

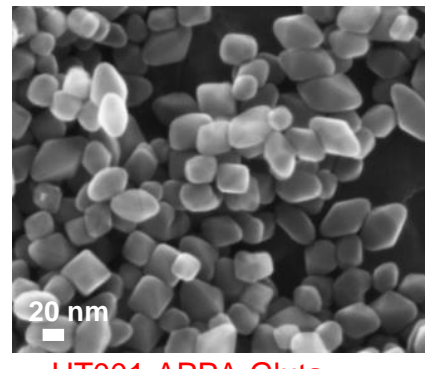

UT001-APPA-Gluta
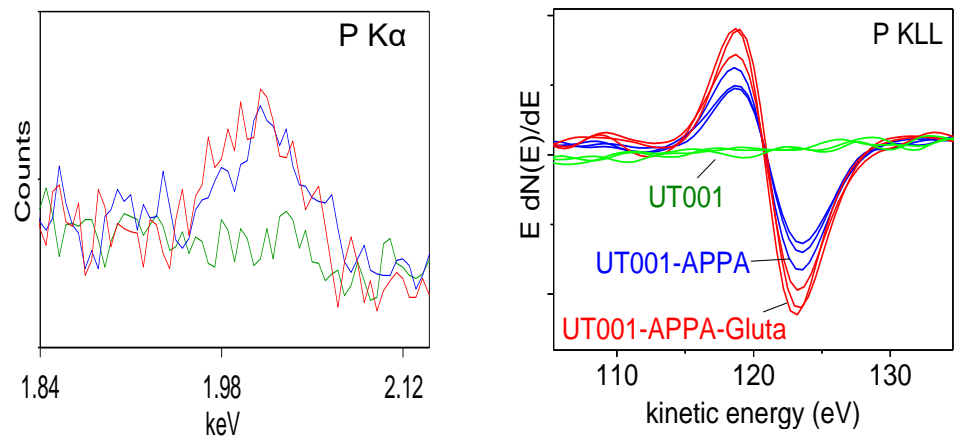

Figure 2. left: high-resolution SEM micrograph of $\mathrm{TiO}_{2} \mathrm{NPs}$ of truncated bipyramidal shape (UT001) after functionalization with firstly APPA and secondly Gluta; middle: $10 \mathrm{kV}$ EDX spectrum window showing P K $\alpha$ of UT001 $\mathrm{TiO}_{2}$ NPs in bare form (green) and after functionalization with APPA (blue) and APPA+Gluta (red); right: differentiated AES spectra of P KLL region of UT001 NP sample before and after functionalization (all three locations on each sample are assigned with the same color). 